\title{
Tetrabutylammonium Iodide-Promoted Thiolation of Oxindoles Using Sulfonyl Chlorides as Sulfenylation Reagents
}

\author{
Xia Zhao ${ }^{1, *}$, Aoqi Wei ${ }^{1}$, Xiaoyu $\mathrm{Lu}^{1}$ and Kui Lu ${ }^{2}$ \\ 1 College of Chemistry, Tianjin Key Laboratory of Structure and Performance for Functional Molecules, \\ Key laboratory of Inorganic-organic Hybrid Functional Material Chemistry, Ministry of Education, \\ Tianjin Normal University, Tianjin 300387, China; weiaoqi920209@163.com (A.W.); \\ 1xy595380904@126.com (X.L.) \\ 2 College of Biotechnology, Tianjin University of Science \& Technology, Tianjin 300457, China; \\ lukui@tust.edu.cn \\ * Correspondence: hxxyzhx@mail.tjnu.edu.cn; Tel.: +86-22-2376-6531
}

Received: 11 July 2017; Accepted: 28 July 2017; Published: 1 August 2017

\begin{abstract}
Sulfanyloxindoles were synthesised by triphenylphosphine-mediated transition-metalfree thiolation of oxindoles using sulfonyl chlorides as sulfenylation reagents. The above reaction was promoted by iodide anions, which was ascribed to the in situ conversion of sulfenyl chlorides into the more reactive sulfenyl iodides. Moreover, the thiolation of 3-aryloxindoles was facilitated by bases. The use of a transition-metal-free protocol, readily available reagents, and mild reaction conditions make this protocol more practical for preparing 3-sulfanyloxindoles than traditional methods.
\end{abstract}

Keywords: thiolation; oxindole; sulfonyl chloride; tetrabutylammonium iodide; triphenylphosphine

\section{Introduction}

Oxindoles and their derivatives have attracted increased attention as a frequently occurring structural motif of both natural products and bioactive compounds [1-5], with thiolation at the C-3 position imparting anticancer [6], antifungal [7], and antitubercular activities (Figure 1) [8]. Therefore, the synthesis of 3-sulfanyloxindoles has been widely investigated, including with methods such as cyclisation of sulfur-containing compounds [9-14], nucleophilic substitution reactions of 3-bromooxindoles (Scheme 1, Equation (1)) [15], electrophilic thiolation of oxindoles with sulfinothioyldibenzene (Scheme 1, Equation (2)) [16] and electrophilic thiolation of oxindoles with $N$-(arylthio)phthalimides (Scheme 1, Equation (3)) [17,18]. Although electrophilic thiolation is the most straightforward method, the need for strongly basic conditions and the limited availability of sulfenylation reagents limit its further application.

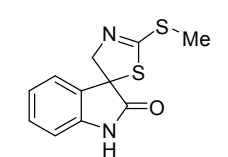

anticancer activity (Ref. 6)

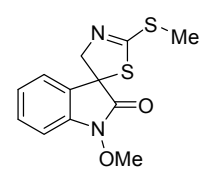

antifungal activity (Ref. 7)

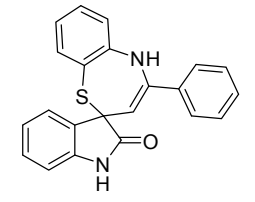

antitubercular activity (Ref. 8)

Figure 1. Bioactive oxindoles with thiolation at the C-3 position.

Recently, the use of sulfonyl chlorides as sulfenylation reagents has been reported by You (Scheme 2, Equation (1)) [19], Zheng (Scheme 2, Equation (2)) [20] and our group (Scheme 2, 
Equations (3) and (4)) [21]. As a part of our on-going development of new sulfenylation methods [21-28], we report here a novel tetrabutylammonium iodide-facilitated thiolation of oxindoles with sulfonyl chlorides as sulfenylation reagents (Scheme 3).

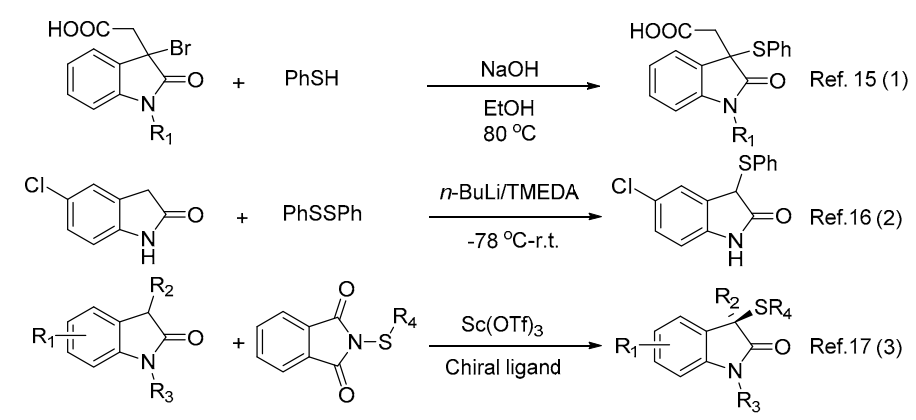

Scheme 1. Previously reported syntheses of 3-sulfanyloxindoles.

$$
\text { (2) }
$$

Scheme 2. Thiolation of electron-rich aromatics using sulfonyl chlorides as sulfenylation reagents.

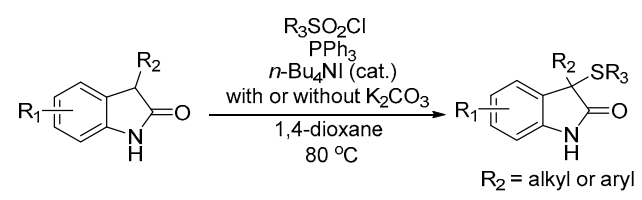

Scheme 3. Present Results for Thiolation of oxindoles by sulfonyl chlorides in the presence of $\mathrm{PPh}_{3}$.

\section{Results}

Treatment of 3-methylindolin-2-one (1a) with 4-methylbenzenesulfonyl chloride (2a) in the presence of $\mathrm{PPh}_{3}$ in 1,4-dioxane at $80{ }^{\circ} \mathrm{C}$ afforded 3-methyl-3-( $p$-tolylthio)indolin-2-one (3aa) in 56\% yield (Table 1, Entry 1). In agreement with our previous studies, this transformation was facilitated by iodide anions [21]. Therefore, a number of classical iodides were initially screened, including potassium iodide $(\mathrm{KI})$, ammonium iodide $\left(\mathrm{NH}_{4} \mathrm{I}\right)$, and tetrabutylammonium iodide $\left(n-\mathrm{Bu}_{4} \mathrm{NI}\right)$, with the highest yield observed for $n$ - $\mathrm{Bu}_{4} \mathrm{NI}$ (Table 1, Entries 2-4). Subsequently, other solvents, such as 1,2-dichloroethane (DCE), toluene, acetonitrile $\left(\mathrm{CH}_{3} \mathrm{CN}\right)$, and $\mathrm{N}, \mathrm{N}$-dimethylformamide (DMF) were tested, but none of them surpassed 1,4-dioxane (Table 1, Entries 5-8). Finally, the effects of temperature and concentration were examined, revealing that decreasing the reaction temperature to $70{ }^{\circ} \mathrm{C}$ or increasing it to $90^{\circ} \mathrm{C}$ diminished the yield (Table 1, Entries 9 and 10), as was also observed for decreasing the concentration of $1 \mathrm{a}$ from $0.5 \mathrm{M}$ to $0.33 \mathrm{M}$ (Table 1, Entry 11). When the concentration 
of 1a was increased from $0.5 \mathrm{M}$ to $1.0 \mathrm{M}$, the desired product was obtained in $86 \%$ yield (Table 1, Entry 12), with further concentration increases leading to diminished yields (Table 1, Entry 13). Notably, increasing the loadings of $\mathbf{2 a}$ and $\mathrm{PPh}_{3}$ to 1.5 and 3.0 equiv., respectively, did not significantly affect the yield (Table 1, Entry 14). Thus, the optimised reaction conditions for the thiolation of 1 a were as follows: 1a $(0.5 \mathrm{mmol}), 2 \mathrm{a}(0.6 \mathrm{mmol}), \mathrm{PPh}_{3}(1.0 \mathrm{mmol}), n$-Bu $\mathrm{BI}_{4}(0.1 \mathrm{mmol})$, and 1,4-dioxane $(0.5 \mathrm{~mL})$ at $80{ }^{\circ} \mathrm{C}$.

Table 1. Optimisation of 3-methylindolin-2-one (1a) thiolation by 4-methylbenzenesulfonyl chloride (2a) in the presence of $\mathrm{PPh}_{3}{ }^{a}$

\begin{tabular}{|c|c|c|c|c|}
\hline & $\frac{1 a}{a d i t i}$ & $\frac{2 a}{2 a}$ & 3аa & V: \\
\hline Entry & Additive/eq. & Temperature $\left({ }^{\circ} \mathrm{C}\right)$ & Solvent/Volume (mL) & Yield $(\%)^{\circ}$ \\
\hline 1 & - & 80 & 1,4-dioxane/1.0 & 56 \\
\hline 2 & $\mathrm{KI} / 0.2$ & 80 & 1,4-dioxane/1.0 & 56 \\
\hline 3 & $\mathrm{NH}_{4} \mathrm{I} / 0.2$ & 80 & 1,4-dioxane/1.0 & 73 \\
\hline 4 & $n-\mathrm{Bu}_{4} \mathrm{NI} / 0.2$ & 80 & 1,4-dioxane/1.0 & 82 \\
\hline 5 & $n-\mathrm{Bu}_{4} \mathrm{NI} / 0.2$ & 80 & $\mathrm{DCE} / 1.0$ & 75 \\
\hline 6 & $n-\mathrm{Bu}_{4} \mathrm{NI} / 0.2$ & 80 & toluene/1.0 & 66 \\
\hline 7 & $n-\mathrm{Bu}_{4} \mathrm{NI} / 0.2$ & 80 & $\mathrm{CH}_{3} \mathrm{CN} / 1.0$ & 46 \\
\hline 8 & $n-\mathrm{Bu}_{4} \mathrm{NI} / 0.2$ & 80 & $\mathrm{DMF} / 1.0$ & 45 \\
\hline 9 & $n-\mathrm{Bu}_{4} \mathrm{NI} / 0.2$ & 70 & 1,4-dioxane/1.0 & 79 \\
\hline 10 & $n-\mathrm{Bu}_{4} \mathrm{NI} / 0.2$ & 90 & 1,4-dioxane/1.0 & 28 \\
\hline 11 & $n-\mathrm{Bu}_{4} \mathrm{NI} / 0.2$ & 80 & 1,4-dioxane/1.5 & 68 \\
\hline 12 & $n-\mathrm{Bu}_{4} \mathrm{NI} / 0.2$ & 80 & 1,4-dioxane/0.5 & 86 \\
\hline 13 & $n-\mathrm{Bu}_{4} \mathrm{NI} / 0.2$ & 80 & 1,4-dioxane/0.3 & 74 \\
\hline 14 & $n-\mathrm{Bu}_{4} \mathrm{NI} / 0.2$ & 80 & 1,4-dioxane/0.5 & $86^{c}$ \\
\hline
\end{tabular}

a Reaction conditions: 1a $(0.5 \mathrm{mmol}), \mathbf{2 a}(0.6 \mathrm{mmol}), \operatorname{PPh} 3(1.0 \mathrm{mmol})$, and additive $(0-0.1 \mathrm{mmol}))$ in an appropriate solvent $(0.3-1.5 \mathrm{~mL})$ for $12 \mathrm{~h}$ at the indicated temperature. ${ }^{\mathrm{b}}$ Yield of product isolated after silica gel chromatography. c $2(0.75 \mathrm{mmol})$ and PPh3 $(1.25 \mathrm{mmol})$ were used.

The optimised conditions were used to investigate the substrate scope of sulfenylation. As shown in Table 2, a series of substituted 3-alkyloxindoles could be coupled with various sulfonyl chlorides to afford the corresponding oxindole thioethers in moderate to excellent yields, with 3-alkyl-(1a, 1c-1g), 3-benzyl-(1h and $\mathbf{1 i})$, and 5-bromo-substituted (1) oxindoles being well tolerated. In the case of aromatic sulfonyl chlorides, both electron-donating and electron-withdrawing groups, as well as diverse ortho-, meta-, and para-substituents (2b-2e) were tolerated. Notably, for electronic effect, aliphatic sulfonyl chlorides ( $2 \mathbf{f}$ and $\mathbf{2 g}$ ) provided the desired thiolation products in a relatively low yield compared with aromatic sulfonyl chlorides.

To further extend the substrate scope of this reaction, we explored the thiolation of 3-aryl-substituted oxindoles with sulfonyl chlorides using 3-(p-tolyl)indolin-2-one (4a) and 3-chlorobenzenesulfonyl chloride (2h) as model substrates in the presence of $\mathrm{PPh}_{3}$ under optimised reaction conditions. However, no desired product (5ah) was obtained (Table 3, Entry 1). Fortunately, when the reaction was carried out at $60^{\circ} \mathrm{C}, 5 \mathrm{ah}$ was obtained in $44 \%$ yield (Table 3, Entry 2). As a further optimisation, potassium carbonate was employed as a base to activate the substrate, affording a significantly improved yield, especially when the reaction was carried out at $40{ }^{\circ} \mathrm{C}$ (Table 3, Entries 5-8). Subsequently, other bases, base loadings, additives, thiolation reagents, and reductants were tested, with the optimal reaction condition identified as: $4 \mathbf{a}(0.25 \mathrm{mmol}), \mathbf{2 h}(0.3 \mathrm{mmol}), \mathrm{PPh}_{3}(0.5 \mathrm{mmol})$, $n$-Bu $\mathrm{Bu}_{4} \mathrm{NI}(0.05 \mathrm{mmol}), \mathrm{K}_{2} \mathrm{CO}_{3}(0.125 \mathrm{mmol})$, and 1,4 -dioxane $(1.0 \mathrm{~mL})$ at $40{ }^{\circ} \mathrm{C}$. 
Table 2. Thiolation of 3-alkyloxindoles with sulfonyl chlorides in the presence of $\mathrm{PPh}_{3} .{ }^{\mathrm{a}}$

\begin{tabular}{|c|c|c|c|c|c|c|c|}
\hline Entry & Oxindole & $\mathbf{R}_{\mathbf{1}}$ & $\mathbf{R}_{\mathbf{2}}$ & $\begin{array}{l}\text { Sulfonyl } \\
\text { Chloride }\end{array}$ & $\mathbf{R}_{\mathbf{3}}$ & Product & Yield (\%) \\
\hline 1 & $1 a$ & $\mathrm{H}$ & $\mathrm{Me}$ & $2 b$ & $p-\mathrm{MeOC}_{6} \mathrm{H}_{4}$ & $3 a b$ & 90 \\
\hline 2 & $1 a$ & $\mathrm{H}$ & $\mathrm{Me}$ & $2 c$ & $m-\mathrm{MeC}_{6} \mathrm{H}_{4}$ & $3 a c$ & 62 \\
\hline 3 & $1 \mathrm{a}$ & $\mathrm{H}$ & $\mathrm{Me}$ & $2 d$ & $3,5-\mathrm{Cl}_{2} \mathrm{C}_{6} \mathrm{H}_{3}$ & 3ad & 68 \\
\hline 4 & $1 a$ & $\mathrm{H}$ & $\mathrm{Me}$ & $2 e$ & $p-\mathrm{BrC}_{6} \mathrm{H}_{4}$ & $3 a e$ & 82 \\
\hline 5 & $1 \mathbf{a}$ & $\mathrm{H}$ & $\mathrm{Me}$ & $2 f$ & cyclopropyl & $3 a f$ & 44 \\
\hline 6 & $1 a$ & $\mathrm{H}$ & $\mathrm{Me}$ & $2 g$ & n-Butyl & $3 a g$ & 56 \\
\hline 7 & $1 b$ & $\mathrm{Br}$ & $\mathrm{Me}$ & $2 a$ & $p-\mathrm{MeC}_{6} \mathrm{H}_{4}$ & $3 \mathbf{b a}$ & 90 \\
\hline 8 & 1c & $\mathrm{H}$ & Et & $2 a$ & $p-\mathrm{MeC}_{6} \mathrm{H}_{4}$ & $3 \mathrm{ca}$ & 79 \\
\hline 9 & 1d & $\mathrm{H}$ & $\operatorname{Pr}$ & $2 a$ & $p-\mathrm{MeC}_{6} \mathrm{H}_{4}$ & $3 d a$ & 81 \\
\hline 10 & 1e & $\mathrm{H}$ & $i-\operatorname{Pr}$ & $2 a$ & $p-\mathrm{MeC}_{6} \mathrm{H}_{4}$ & 3ea & $63^{b}$ \\
\hline 11 & 1f & $\mathrm{H}$ & $i-\mathrm{Bu}$ & $2 a$ & $p-\mathrm{MeC}_{6} \mathrm{H}_{4}$ & $3 \mathrm{fa}$ & $78^{b}$ \\
\hline 12 & $1 \mathrm{~g}$ & $\mathrm{H}$ & cyclohexyl & $2 a$ & $p-\mathrm{MeC}_{6} \mathrm{H}_{4}$ & 3ga & $67^{b}$ \\
\hline 13 & $1 \mathrm{~g}$ & $\mathrm{H}$ & cyclohexyl & $2 b$ & $p-\mathrm{MeOC}_{6} \mathrm{H}_{4}$ & $3 g b$ & $65^{b}$ \\
\hline 14 & 1h & $\mathrm{H}$ & $p-\mathrm{NCC}_{6} \mathrm{H}_{4} \mathrm{CH}_{2}$ & $2 a$ & $p-\mathrm{MeC}_{6} \mathrm{H}_{4}$ & 3ha & $60^{b}$ \\
\hline 15 & $\mathbf{1 i}$ & $\mathrm{H}$ & $p-\mathrm{ClC}_{6} \mathrm{H}_{4} \mathrm{CH}_{2}$ & $2 a$ & $p-\mathrm{MeC}_{6} \mathrm{H}_{4}$ & $3 i a$ & $79^{b}$ \\
\hline
\end{tabular}

a Reaction conditions: 1a-1d $(0.5 \mathrm{mmol}), \mathbf{2 a}-\mathbf{2 g}(0.6 \mathrm{mmol})$, PPh3 $(1.0 \mathrm{mmol}), n$-Bu4NI $(0.1 \mathrm{mmol}), 1$, 4-dioxane $(0.5 \mathrm{~mL}), 80^{\circ} \mathrm{C}, 12 \mathrm{~h} .{ }^{\mathrm{b}} \mathbf{1 e}-1 \mathrm{i}(0.25 \mathrm{mmol}), \mathbf{2 a}-\mathbf{2 g}(0.3 \mathrm{mmol})$, PPh3 (1.5 mmol), $n$-Bu4NI (0.05 mmol), 1,4-dioxane $(0.25 \mathrm{~mL}), 80^{\circ} \mathrm{C}, 6-30 \mathrm{~h}$.

Table 3. Optimisation of 3-( $p$-tolyl)indolin-2-one (4a) thiolation by 3-chlorobenzenesulfonyl chloride (2h) in the presence of $\mathrm{PPh}_{3}{ }^{\mathrm{a}}$
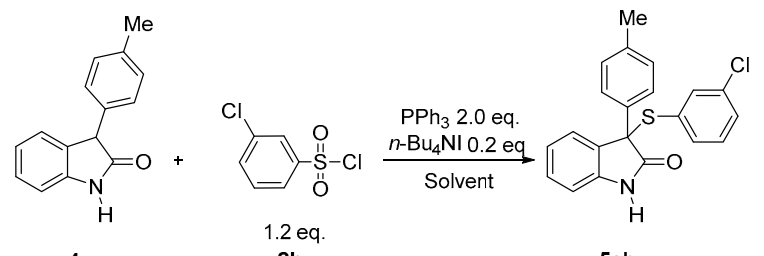

4a

$2 \mathrm{~h}$

$5 \mathrm{ah}$

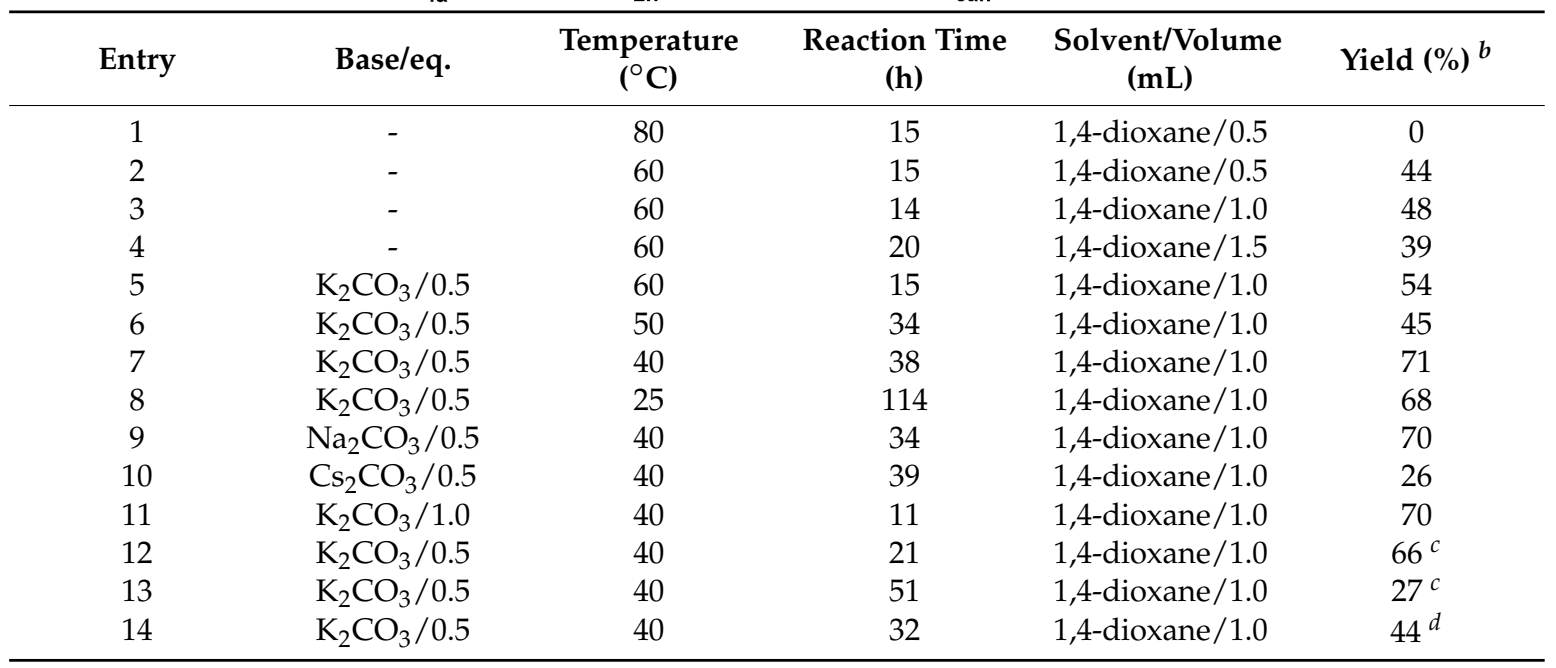

a Reaction conditions: $4 \mathrm{a}(0.25 \mathrm{mmol}), 2 \mathrm{~h}$ ( $0.3 \mathrm{mmol})$, PPh3 $(0.5 \mathrm{mmol})$, and $n$-Bu4NI $(0.05 \mathrm{mmol})$ in 1,4 -dioxane $(0.5-1.5 \mathrm{~mL})$ for indicated time and at specified temperature. b Yield of product isolated after silica gel chromatography. ${ }^{c}$-Bu4NI $(0.125 \mathrm{mmol})$ was used. d2h $(0.375 \mathrm{mmol})$ and PPh3 $(0.75 \mathrm{mmol})$ were used. 
With the new optimised conditions in hand, the generality of the thiolation reaction was examined using various 3-aryloxindoles and arylsulfonyl chlorides (Figure 2), with the desired sulfenylation products (5aa-5ca) obtained in moderate yields.<smiles>Cc1ccc(SC2(c3ccc(C)cc3)C(=O)Nc3ccccc32)cc1</smiles>

5aa, $42 \%, 48 h$

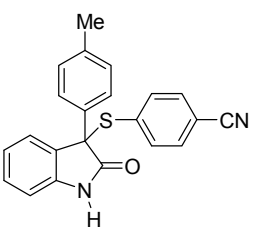

5ai, $41 \%, 48 \mathrm{~h}$

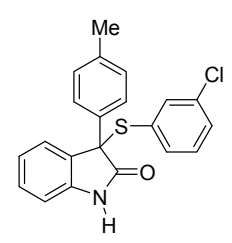

5ah, $71 \%, 48 \mathrm{~h}$<smiles>Cc1ccc(SC2(c3ccc(C)cc3)C(=O)Nc3ccc(Br)cc32)cc1</smiles>

$\mathrm{MeO}$<smiles>COc1cccc(SC2(c3ccc(C)cc3)C(=O)Nc3ccccc32)c1</smiles>

5 ca, $39 \%, 48 \mathrm{~h}$

${ }^{a}$ Reaction conditions: 4 (0.25 mmol), 2 (0.3 mmol), $\mathrm{PPh}_{3}(0.5 \mathrm{mmol}), \mathrm{K}_{2} \mathrm{CO}_{3}(0.125$ $\mathrm{mmol})$, and $n-\mathrm{Bu}_{4} \mathrm{NI}(0.05 \mathrm{mmol})$ in 1,4 -dioxane $(1.0 \mathrm{~mL})$ for $48-57 \mathrm{~h}$ and $a 0^{\circ} \mathrm{C}$.

Figure 2. Thiolation of 3-aryloxindoles with sulfonyl chlorides in the presence of $\mathrm{PPh}_{3} .{ }^{\mathrm{a}}$

\section{Discussion}

Based on our previous work [21], a plausible reaction mechanism was proposed (Scheme 4), featuring the initial reduction of sulfonyl chloride 2 by $\mathrm{PPh}_{3}$ to sulfenyl chloride $\mathbf{F}$ via intermediates A-E. F is converted into sulfenyl iodide $\mathbf{G}$ in the presence of iodide anions. Finally, electrophilic thiolation of oxindoles $\mathbf{1}$ by $\mathbf{G}$ gives the corresponding oxindole thioethers.

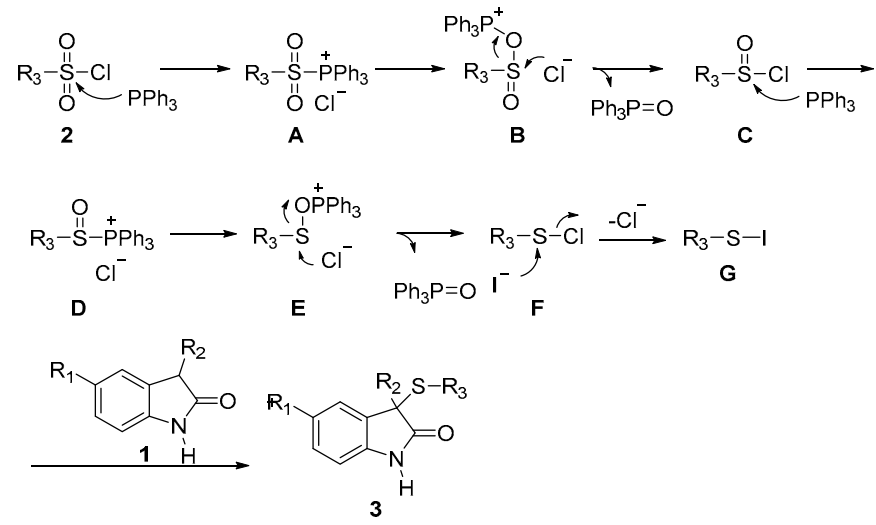

Scheme 4. Proposed reaction mechanism.

\section{Materials and Methods}

\subsection{General Methods and Material}

All solvents were distilled prior to use. Unless otherwise noted, chemicals were used as received without further purification. For chromatography, 200-300 mesh silica gel was employed. ${ }^{1} \mathrm{H}$ - and ${ }^{13} \mathrm{C}-\mathrm{NMR}$ spectra were recorded at $400 \mathrm{MHz}$ and $100 \mathrm{MHz}$ respectively. Chemical shifts are reported in ppm using tetramethylsilane as internal standard (see supplementary). HRMS was performed on an FTMS mass instrument. Melting points are reported as uncorrected.

\subsection{Synthesis of Oxindoles}

1c-1i were synthesized according to the literature procedures [29]. 


\subsubsection{5-Bromo-3-methylindolin-2-one (1b)}

3-methylindolin-2-one $(441 \mathrm{mg}, 3 \mathrm{mmol})$ in acetonitrile $(5 \mathrm{~mL})$ was cooled to $-15^{\circ} \mathrm{C}$. $\mathrm{NBS}(534 \mathrm{mg}$, $3 \mathrm{mmol})$ was added. After stirring for $1 \mathrm{~h}$, the reaction was diluted with water $(10 \mathrm{~mL})$ and extracted with EtOAc $(20 \mathrm{~mL})$ for three times. The combined organic phase was washed with brine, dried over $\mathrm{Na}_{2} \mathrm{SO}_{4}$ and concentrated under reduced pressure to give a residue which was purified by silica gel column chromatography to afford compound $\mathbf{1 b}(454 \mathrm{mg}, 67 \%)$ as a white solid.

\subsubsection{3-(p-Tolyl)indolin-2-one (4a)}

Indoline-2,3-dione $(1.47 \mathrm{~g}, 10 \mathrm{mmol})$ in THF $(20 \mathrm{~mL})$ was cooled to $-15^{\circ} \mathrm{C}$. $\mathrm{NaH}(60 \% / \mathrm{mineral}$ oil, $600 \mathrm{mg}, 15 \mathrm{mmol})$ was added. After stirring for $30 \mathrm{~min}, p$-tolylmagnesium bromide $(1.0 \mathrm{M} / \mathrm{THF}, 10 \mathrm{~mL}$, $10 \mathrm{mmol}$ ) was added. The reaction mixture was allowed to warm to room temperature and stirred for $1 \mathrm{~h}$. Then the reaction was quenched with $\mathrm{NH} 4 \mathrm{Cl}(\mathrm{aq})(30 \mathrm{~mL})$ and extracted with $\mathrm{Et}_{2} \mathrm{O}(50 \mathrm{~mL})$ for three times. After stirring for $1 \mathrm{~h}$, the reaction was diluted with water $(10 \mathrm{~mL})$ and extracted with EtOAc $(20 \mathrm{~mL})$ three times. The combined organic phase was washed with brine, dried over $\mathrm{Na}_{2} \mathrm{SO}_{4}$ and concentrated under reduced pressure to give a residue, which was purified by silica gel column chromatography to afford compound $\mathbf{1 b}(454 \mathrm{mg}, 67 \%)$ as a yellow solid.

\subsection{General Procedure for the Synthesis of 3aa, 3ab, 3ac, 3ad, 3ae, 3af, 3ag, 3ba, 3ca and 3da}

Oxindole (0.5 mmol), sulfonyl chloride $(0.6 \mathrm{mmol}), \mathrm{PPh}_{3}(1.0 \mathrm{mmol}), n-\mathrm{Bu}_{4} \mathrm{NI}(0.1 \mathrm{mmol})$ and dry 1,4-dioxane $(0.5 \mathrm{~mL})$ were mixed in an oven dried sealed tube. The mixture was stirred at $80{ }^{\circ} \mathrm{C}$ for $12 \mathrm{~h}$. Then, the solvent was evaporated under reduced pressure and the residue was purified by silica gel column chromatography (PE:EA $=5: 1$ or PE:EA $=3: 1$ ) to afford the pure product.

\subsection{General Procedure for the Synthesis of $3 \mathbf{e a}, 3 \mathbf{f a}, 3 \mathbf{g a}, 3 \mathbf{g b}$, 3ha and 3ia}

Oxindole $(0.25 \mathrm{mmol})$, sulfonyl chloride $(0.3 \mathrm{mmol}), \mathrm{PPh}_{3}(0.5 \mathrm{mmol}), n-\mathrm{Bu}_{4} \mathrm{NI}(0.05 \mathrm{mmol})$ and dry 1,4-dioxane $(0.25 \mathrm{~mL})$ were mixed in an oven dried sealed tube. The mixture was stirred at $80{ }^{\circ} \mathrm{C}$ for 6-30 h. Then, the solvent was evaporated under reduced pressure and the residue was purified by silica gel column chromatography (PE:EA = 5:1, PE:EA = 4:1 or PE:EA = 3:1) to afford the pure product.

\subsection{General Procedure for the Synthesis of 5aa, 5ai, 5ah, 5ba and 5ca}

Oxindole (0.25 mmol), sulfonyl chloride (0.3 mmol), $\mathrm{PPh}_{3}(0.5 \mathrm{mmol}), n-\mathrm{Bu}_{4} \mathrm{NI}(0.05 \mathrm{mmol}), \mathrm{K}_{2} \mathrm{CO}_{3}$ $(0.125 \mathrm{mmol})$ and dry 1,4-dioxane $(1.0 \mathrm{~mL})$ were mixed in an oven-dried sealed tube. The mixture was stirred at $40{ }^{\circ} \mathrm{C}$ for the time indicated. Then, the solvent was evaporated under reduced pressure and the residue was purified by silica gel column chromatography (PE:EA $=5: 1$ or PE:EA = 3:1) to afford the pure product.

3-Methyl-3-( $p$-tolylthio)indolin-2-one (3aa). After purification by silica gel column chromatography (PE:EA = 5:1), compound 3aa was isolated as a white solid $(116 \mathrm{mg}, 86 \%)$; m.p. $=151-152{ }^{\circ} \mathrm{C} ; R_{\mathrm{f}}$ $(\mathrm{PE}: \mathrm{EA}=3: 1)=0.32 ;{ }^{1} \mathrm{H}-\mathrm{NMR}\left(400 \mathrm{MHz}, \mathrm{CDCl}_{3}\right): \delta 8.39(\mathrm{~s}, 1 \mathrm{H}), 7.35(\mathrm{~d}, J=7.4 \mathrm{~Hz}, 1 \mathrm{H}), 7.15(\mathrm{td}, J=7.7$ $\mathrm{Hz}, 1.3 \mathrm{~Hz}, 1 \mathrm{H}), 7.11(\mathrm{~d}, J=8.0 \mathrm{~Hz}, 2 \mathrm{H}), 7.07(\mathrm{td}, J=7.5 \mathrm{~Hz}, 1.0 \mathrm{~Hz}, 1 \mathrm{H}), 6.91(\mathrm{~d}, J=7.9 \mathrm{~Hz}, 2 \mathrm{H}), 6.70$ $(\mathrm{d}, J=7.7 \mathrm{~Hz}, 1 \mathrm{H}), 2.24$ (s, 3H), 1.69 (s, 3H); ${ }^{13} \mathrm{C}-\mathrm{NMR}\left(100 \mathrm{MHz}, \mathrm{CDCl}_{3}\right): \delta 179.3,139.8,139.6,136.3$, 132.1, 129.2, 128.6, 126.4, 124.2, 122.6, 109.7, 54.9, 21.4, 21.2; HRMS (ESI) m/e calcd. for $\mathrm{C}_{16} \mathrm{H}_{15} \mathrm{NOS}$ $(\mathrm{M}+\mathrm{H})^{+}$270.0947, found 270.0947 .

3-[(4-Methoxyphenyl)thio]-3-methylindolin-2-one (3ab). After purification by silica gel column chromatography (PE:EA = 3:1), compound 3ab was isolated as a pink solid (128 $\mathrm{mg}$, 90\%); m.p. $=153-154{ }^{\circ} \mathrm{C} ; R_{\mathrm{f}}(\mathrm{PE}: \mathrm{EA}=3: 1)=0.27 ;{ }^{1} \mathrm{H}-\mathrm{NMR}\left(400 \mathrm{MHz}, \mathrm{CDCl}_{3}\right): \delta 7.67(\mathrm{~s}, 1 \mathrm{H}), 7.36(\mathrm{~d}, J=7.4 \mathrm{~Hz}$, $1 \mathrm{H}), 7.17-7.13(\mathrm{~m}, 3 \mathrm{H}), 7.07(\mathrm{td}, J=7.6 \mathrm{~Hz}, 1.0 \mathrm{~Hz}, 1 \mathrm{H}), 6.67-6.62(\mathrm{~m}, 3 \mathrm{H}), 3.72(\mathrm{~s}, 3 \mathrm{H}), 1.69$ (s, 3H); ${ }^{13} \mathrm{C}-\mathrm{NMR}\left(100 \mathrm{MHz}_{,} \mathrm{CDCl}_{3}\right): \delta 179.6,160.7,140.0,137.9,132.1,128.6,124.1,122.6,120.7,113.9,109.8$, 55.1, 55.1, 21.1; HRMS (ESI) m/e calcd. for $\mathrm{C}_{16} \mathrm{H}_{15} \mathrm{NO} 2 \mathrm{~S}(\mathrm{M}+\mathrm{H})^{+} 286.0896$, found 286.0896. 
3-Methyl-3-( $m$-tolylthio)indolin-2-one (3ac). After purification by silica gel column chromatography (PE:EA = 5:1), compound 3ac was isolated as a pale solid $(83 \mathrm{mg}, 62 \%)$; m.p. $=106-107{ }^{\circ} \mathrm{C}$; $R_{\mathrm{f}}(\mathrm{PE}: \mathrm{EA}=3: 1)=0.45 ;{ }^{1} \mathrm{H}-\mathrm{NMR}\left(400 \mathrm{MHz}, \mathrm{CDCl}_{3}\right): \delta 7.71(\mathrm{~s}, 1 \mathrm{H}), 7.22(\mathrm{t}, J=7.2 \mathrm{~Hz}, 2 \mathrm{H}), 7.17-7.09$ $(\mathrm{m}, 3 \mathrm{H}), 7.02(\mathrm{t}, J=7.5 \mathrm{~Hz}, 1 \mathrm{H}), 6.94(\mathrm{td}, J=7.6 \mathrm{~Hz}, 1.1 \mathrm{~Hz}, 1 \mathrm{H}), 6.69(\mathrm{~d}, J=7.7 \mathrm{~Hz}, 1 \mathrm{H}), 2.30(\mathrm{~s}, 3 \mathrm{H})$, $1.74(\mathrm{~s}, 3 \mathrm{H}) ;{ }^{13} \mathrm{C}-\mathrm{NMR}\left(100 \mathrm{MHz}, \mathrm{CDCl}_{3}\right): \delta 179.6,143.7,139.9,137.5,131.9,130.2,129.6,129.4,128.7$, 125.7, 124.1, 122.4, 109.9, 55.0, 21.7, 21.0; HRMS (ESI) m/e calcd. for $\mathrm{C}_{16} \mathrm{H}_{15} \mathrm{NOS}(\mathrm{M}+\mathrm{H})^{+}$270.0947, found 270.0946 .

3-((3,5-Dichlorophenyl)thio)-3-methylindolin-2-one (3ad). After purification by silica gel column chromatography (PE:EA = 5:1), compound 3ad was isolated as a white solid $(110 \mathrm{mg}, 68 \%$ ); m.p. $=176-177{ }^{\circ} \mathrm{C} ; R_{\mathrm{f}}(\mathrm{PE}: \mathrm{EA}=5: 1)=0.30 ;{ }^{1} \mathrm{H}-\mathrm{NMR}\left(400 \mathrm{MHz}, d_{6}\right.$-DMSO): $\delta 10.50(\mathrm{~s}, 1 \mathrm{H}), 7.56$ $(\mathrm{s}, 1 \mathrm{H}), 7.38(\mathrm{~d}, J=7.4 \mathrm{~Hz}, 1 \mathrm{H}), 7.18(\mathrm{t}, J=7.6 \mathrm{~Hz}, 1 \mathrm{H}), 7.10(\mathrm{~d}, J=1.8 \mathrm{~Hz}, 2 \mathrm{H}), 7.03(\mathrm{t}, J=7.5 \mathrm{~Hz}, 1 \mathrm{H})$, $6.71(\mathrm{~d}, J=7.7 \mathrm{~Hz}, 1 \mathrm{H}), 1.58(\mathrm{~s}, 3 \mathrm{H}) ;{ }^{13} \mathrm{C}-\mathrm{NMR}\left(100 \mathrm{MHz}, d_{6}\right.$-DMSO): $\delta 177.0,141.1,133.9,133.6,133.1$, $130.7,129.3,129.0,124.1,122.1,109.7,54.8,21.3$; HRMS (ESI) m/e calcd. for $\mathrm{C}_{15} \mathrm{H}_{11} \mathrm{Cl}_{2} \mathrm{NOS}(\mathrm{M}+\mathrm{H})^{+}$ 324.0011, found 324.0010.

3-((4-Bromophenyl)thio)-3-methylindolin-2-one (3ae). After purification by silica gel column chromatography (PE:EA = 5:1), compound 3ae was isolated as a white solid $(136 \mathrm{mg}, 82 \%)$; m.p. $=135-137^{\circ} \mathrm{C} ; R_{\mathrm{f}}(\mathrm{PE}: \mathrm{EA}=3: 1)=0.40 ;{ }^{1} \mathrm{H}-\mathrm{NMR}\left(400 \mathrm{MHz}, \mathrm{CDCl}_{3}\right): \delta 7.65(\mathrm{~s}, 1 \mathrm{H}), 7.38(\mathrm{~d}, J=7.4 \mathrm{~Hz}$, $1 \mathrm{H}), 7.25-7.23(\mathrm{~m}, 2 \mathrm{H}), 7.17(\mathrm{td}, J=7.7 \mathrm{~Hz}, 1.3 \mathrm{~Hz}, 1 \mathrm{H}), 7.11-7.07(\mathrm{~m}, 3 \mathrm{H}), 6.68(\mathrm{~d}, J=7.7 \mathrm{~Hz}, 1 \mathrm{H}), 1.70$ (s, 3H); ${ }^{13} \mathrm{C}-\mathrm{NMR}\left(100 \mathrm{MHz}, \mathrm{CDCl}_{3}\right): \delta 179.0,139.8,137.7,131.7,131.6,129.0,128.9,124.4,124.2,122.8$, 110.0, 55.1, 21.5; HRMS (ESI) m/e calcd. for $\mathrm{C}_{15} \mathrm{H}_{12} \operatorname{BrNOS}(\mathrm{M}+\mathrm{H})^{+} 333.9895$, found 333.9895.

3-(Cyclopropylthio)-3-methylindolin-2-one (3af). After purification by silica gel column chromatography (PE:EA $=5: 1)$, compound 3af was isolated as a white solid $(48 \mathrm{mg}, 44 \%$ ); m.p. $=122-124{ }^{\circ} \mathrm{C} ; R_{\mathrm{f}}(\mathrm{PE}: \mathrm{EA}=3: 1)=0.30 ;{ }^{1} \mathrm{H}-\mathrm{NMR}\left(400 \mathrm{MHz}, \mathrm{CDCl}_{3}\right): \delta 9.57(\mathrm{~s}, 1 \mathrm{H}), 7.34(\mathrm{~d}, J=7.4 \mathrm{~Hz}$, $1 \mathrm{H}), 7.24(\mathrm{td}, J=7.6 \mathrm{~Hz}, 0.8 \mathrm{~Hz}, 1 \mathrm{H}), 7.08(\mathrm{t}, J=7.5 \mathrm{~Hz}, 1 \mathrm{H}), 6.98(\mathrm{~d}, J=7.7 \mathrm{~Hz}, 1 \mathrm{H}), 1.67(\mathrm{~s}, 3 \mathrm{H})$, $1.62-1.56(\mathrm{~s}, 1 \mathrm{H}), 0.72-0.66(\mathrm{~s}, 1 \mathrm{H}), 0.63-0.51(\mathrm{~m}, 2 \mathrm{H}), 0.35-0.28(\mathrm{~m}, 1 \mathrm{H}) ;{ }^{13} \mathrm{C}-\mathrm{NMR}\left(100 \mathrm{MHz}, \mathrm{CDCl}_{3}\right)$ : $\delta 181.3,140.1,132.5,128.6,123.9,122.8,110.1,52.5,21.9,10.1,7.53,5.65$; HRMS (ESI) $m / e$ calcd. for $\mathrm{C}_{12} \mathrm{H}_{13} \mathrm{NOS}(\mathrm{M}+\mathrm{H})^{+} 220.0790$, found 220.0789 .

3-(Butylthio)-3-methylindolin-2-one (3ag). After purification by silica gel column chromatography (PE:EA = 5:1), compound 3ag was isolated as a yellow liquid $(66 \mathrm{mg}, 56 \%) ; R_{\mathrm{f}}(\mathrm{PE}: \mathrm{EA}=3: 1)=0.42$; ${ }^{1} \mathrm{H}-\mathrm{NMR}\left(400 \mathrm{MHz}, \mathrm{CDCl}_{3}\right): \delta 8.67(\mathrm{~s}, 1 \mathrm{H}), 7.33(\mathrm{~d}, J=7.4 \mathrm{~Hz}, 1 \mathrm{H}), 7.23(\mathrm{td}, J=7.7 \mathrm{~Hz}, 1.2 \mathrm{~Hz}, 1 \mathrm{H}), 7.09$ $(\mathrm{td}, J=7.6 \mathrm{~Hz}, 0.7 \mathrm{~Hz}, 1 \mathrm{H}), 6.93(\mathrm{~d}, J=7.7 \mathrm{~Hz}, 1 \mathrm{H}), 2.44(\mathrm{dt}, J=11.6 \mathrm{~Hz}, 7.3 \mathrm{~Hz}, 1 \mathrm{H}), 2.28(\mathrm{dt}, J=11.6 \mathrm{~Hz}$, $7.4 \mathrm{~Hz}, 1 \mathrm{H}), 1.67(\mathrm{~s}, 3 \mathrm{H}), 1.41-1.36(\mathrm{~m}, 2 \mathrm{H}), 1.32-1.25(\mathrm{~m}, 2 \mathrm{H}), 0.79(\mathrm{t}, J=7.3 \mathrm{~Hz}, 3 \mathrm{H}) ;{ }^{13} \mathrm{C}-\mathrm{NMR}$ (100 MHz, $\mathrm{CDCl}_{3}$ ): $\delta$ 180.1, 139.7, 132.3, 128.7, 124.0, 123.0, 109.8, 50.8, 30.8, 28.8, 22.4, 22.0, 13.5; HRMS (ESI) $m / e$ calcd. for $\mathrm{C}_{13} \mathrm{H}_{17} \mathrm{NOS}(\mathrm{M}+\mathrm{H})^{+} 236.1103$, found 236.1103 .

5-Bromo-3-methyl-3-(p-tolylthio)indolin-2-one (3ba). After purification by silica gel column chromatography (PE:EA = 3:1), compound 3ba was isolated as a pale solid $(156 \mathrm{mg}, 90 \%)$; m.p. $=167-168{ }^{\circ} \mathrm{C} ; R_{\mathrm{f}}(\mathrm{PE}: \mathrm{EA}=3: 1)=0.33 ;{ }^{1} \mathrm{H}-\mathrm{NMR}\left(400 \mathrm{MHz}, \mathrm{CDCl}_{3}\right): \delta 8.58(\mathrm{~s}, 1 \mathrm{H}), 7.43(\mathrm{~d}, J=1.9 \mathrm{~Hz}, 1 \mathrm{H})$, $7.28(\mathrm{dd}, J=8.2 \mathrm{~Hz}, J=2.0 \mathrm{~Hz}, 1 \mathrm{H}), 7.12(\mathrm{~d}, J=8.1 \mathrm{~Hz}, 2 \mathrm{H}), 6.95(\mathrm{~d}, J=7.9 \mathrm{~Hz}, 2 \mathrm{H}), 6.60(\mathrm{~d}, J=8.3 \mathrm{~Hz}$, $1 \mathrm{H}), 2.26(\mathrm{~s}, 3 \mathrm{H}), 1.68(\mathrm{~s}, 3 \mathrm{H}) ;{ }^{13} \mathrm{C}-\mathrm{NMR}\left(100 \mathrm{MHz}, \mathrm{CDCl}_{3}\right): \delta 179.6,139.9,138.9,136.2,134.1,131.4$, 129.3, 127.2, 125.8, 115.1, 111.5, 55.0, 21.2, 21.2; HRMS (ESI) m/e calcd. for $\mathrm{C}_{16} \mathrm{H}_{14} \mathrm{BrNOS}(\mathrm{M}+\mathrm{H})^{+}$ 348.0052 , found 348.0052 .

3-Ethyl-3-(p-tolylthio)indolin-2-one (3ca). After purification by silica gel column chromatography (PE:EA $=5: 1)$, compound 3ca was isolated as a white solid $(106 \mathrm{mg}, 79 \%) ; \mathrm{m} . \mathrm{p} .=178-179{ }^{\circ} \mathrm{C} ; R_{\mathrm{f}}(\mathrm{PE}: \mathrm{EA}$ $=3: 1)=0.37 ;{ }^{1} \mathrm{H}-\mathrm{NMR}\left(400 \mathrm{MHz}, \mathrm{CDCl}_{3}\right): \delta 7.91(\mathrm{~s}, 1 \mathrm{H}), 7.32(\mathrm{~d}, J=7.4 \mathrm{~Hz}, 1 \mathrm{H}), 7.15(\mathrm{td}, J=7.6 \mathrm{~Hz}$, $1.3 \mathrm{~Hz}, 1 \mathrm{H}), 7.12(\mathrm{~d}, J=8.1 \mathrm{~Hz}, 2 \mathrm{H}), 7.07(\mathrm{td}, J=7.5 \mathrm{~Hz}, 1.0 \mathrm{~Hz}, 1 \mathrm{H}), 6.91(\mathrm{~d}, J=7.9 \mathrm{~Hz}, 2 \mathrm{H}), 6.67$ $(\mathrm{d}, J=7.7 \mathrm{~Hz}, 1 \mathrm{H}), 2.24(\mathrm{~s}, 3 \mathrm{H}), 2.23-2.09(\mathrm{~m}, 2 \mathrm{H}), 0.76(\mathrm{t}, J=7.4 \mathrm{~Hz}, 3 \mathrm{H}) ;{ }^{13} \mathrm{C}-\mathrm{NMR}\left(100 \mathrm{MHz}, \mathrm{CDCl}_{3}\right)$ : $\delta 179.0,140.8,139.5,136.4,123.0,129.1,128.5,126.0,124.4,122.5,109.8,60.0,28.5,21.2,9.23$; HRMS (ESI) m/e calcd. for $\mathrm{C}_{17} \mathrm{H}_{17} \mathrm{NOS}(\mathrm{M}+\mathrm{H})^{+} 284.1103$, found 284.1105 . 
3-Propyl-3-( $p$-tolylthio)indolin-2-one (3da). After purification by silica gel column chromatography (PE:EA = 5:1), compound 3da was isolated as a pale solid $(74 \mathrm{mg}, 81 \%) ; \mathrm{m} . \mathrm{p} .=152-153{ }^{\circ} \mathrm{C} ; R_{\mathrm{f}}$ (PE:EA $=3: 1)=0.40 ;{ }^{1} \mathrm{H}-\mathrm{NMR}\left(400 \mathrm{MHz}, \mathrm{CDCl}_{3}\right): \delta 8.67(\mathrm{~s}, 1 \mathrm{H}), 7.32(\mathrm{~d}, J=7.3 \mathrm{~Hz}, 1 \mathrm{H}), 7.15(\mathrm{td}, J=7.6 \mathrm{~Hz}$, $1.3 \mathrm{~Hz}, 1 \mathrm{H}), 7.10(\mathrm{~d}, J=8.1 \mathrm{~Hz}, 2 \mathrm{H}), 7.06(\mathrm{td}, J=7.5 \mathrm{~Hz}, 0.8 \mathrm{~Hz}, 1 \mathrm{H}), 6.89(\mathrm{~d}, J=7.9 \mathrm{~Hz}, 2 \mathrm{H}), 6.70$ $(\mathrm{d}, J=7.6 \mathrm{~Hz}, 1 \mathrm{H}), 2.22(\mathrm{~s}, 3 \mathrm{H}), 2.17-2.01(\mathrm{~m}, 2 \mathrm{H}), 1.20-1.05(\mathrm{~m}, 2 \mathrm{H}), 0.84(\mathrm{t}, J=7.3 \mathrm{~Hz}, 3 \mathrm{H}) ;{ }^{13} \mathrm{C}-\mathrm{NMR}$ $\left(100 \mathrm{MHz}, \mathrm{CDCl}_{3}\right): \delta 178.9,140.6,139.5,136.4,130.4,129.1,128.5,126.0,124.5,122.5,109.7,59.4,37.4$, 21.2, 18.3, 14.0; HRMS (ESI) $m / e$ calcd. for $\mathrm{C}_{18} \mathrm{H}_{19} \mathrm{NOS}(\mathrm{M}+\mathrm{H})^{+} 298.1260$, found 298.1267.

3-Isopropyl-3-( $p$-tolylthio)indolin-2-one (3ea). After purification by silica gel column chromatography (PE:EA = 5:1), compound 3ea was isolated as a white solid $(47 \mathrm{mg}, 63 \%)$; m.p. $=162-163{ }^{\circ} \mathrm{C}$; $R_{\mathrm{f}}(\mathrm{PE}: \mathrm{EA}=5: 1)=0.32 ;{ }^{1} \mathrm{H}-\mathrm{NMR}\left(400 \mathrm{MHz}, \mathrm{CDCl}_{3}\right): \delta 8.45(\mathrm{~s}, 1 \mathrm{H}), 7.44(\mathrm{~d}, J=7.4 \mathrm{~Hz}, 1 \mathrm{H}), 7.14$ $(\mathrm{td}, J=7.6 \mathrm{~Hz}, 1.2 \mathrm{~Hz}, 1 \mathrm{H}), 7.07-7.03(\mathrm{~m}, 3 \mathrm{H}), 6.85(\mathrm{~d}, J=7.8 \mathrm{~Hz}, 2 \mathrm{H}), 6.66(\mathrm{~d}, J=7.6 \mathrm{~Hz}, 1 \mathrm{H}), 2.47$ $(\mathrm{h}, J=6.8 \mathrm{~Hz}, 1 \mathrm{H}), 2.20(\mathrm{~s}, 3 \mathrm{H}), 1.28(\mathrm{~d}, J=7.0 \mathrm{~Hz}, 3 \mathrm{H}), 0.87(\mathrm{~d}, J=6.8 \mathrm{~Hz}, 3 \mathrm{H}) ;{ }^{13} \mathrm{C}-\mathrm{NMR}(100 \mathrm{MHz}$, $\left.\mathrm{CDCl}_{3}\right): \delta 179.2,140.8,139.3,136.2,129.2,129.1,128.4,126.0,125.5,122.2,109.7,64.1,33.8,21.1,18.0$, 17.7; HRMS (ESI) $m / e$ calcd. for $\mathrm{C}_{18} \mathrm{H}_{19} \mathrm{NOS}(\mathrm{M}+\mathrm{H})^{+} 298.1260$, found 298.1259.

3-Isopentyl-3-( $p$-tolylthio)indolin-2-one (3fa). After purification by silica gel column chromatography (PE:EA = 5:1), compound 3fa was isolated as a white solid $(64 \mathrm{mg}, 78 \%)$; m.p. $=159-160{ }^{\circ} \mathrm{C}$; $R_{\mathrm{f}}(\mathrm{PE}: \mathrm{EA}=5: 1)=0.30 ;{ }^{1} \mathrm{H}-\mathrm{NMR}\left(400 \mathrm{MHz}, \mathrm{CDCl}_{3}\right): \delta 7.91(\mathrm{~s}, 1 \mathrm{H}), 7.32(\mathrm{~d}, J=7.3 \mathrm{~Hz}, 1 \mathrm{H}), 7.14$ $(\mathrm{td}, J=7.6 \mathrm{~Hz}, 1.2 \mathrm{~Hz}, 1 \mathrm{H}), 7.10-7.05(\mathrm{~m}, 3 \mathrm{H}), 6.90(\mathrm{~d}, J=7.9 \mathrm{~Hz}, 2 \mathrm{H}), 6.65(\mathrm{~d}, J=7.6 \mathrm{~Hz}, 1 \mathrm{H}), 2.23$ $(\mathrm{s}, 3 \mathrm{H}), 2.20-2.04(\mathrm{~m}, 2 \mathrm{H}), 1.51-1.44(\mathrm{~m}, 1 \mathrm{H}), 1.09-0.86(\mathrm{~m}, 2 \mathrm{H}), 0.81(\mathrm{~d}, J=6.6 \mathrm{~Hz}, 6 \mathrm{H}), 0.80(\mathrm{~s}, 3 \mathrm{H})$; ${ }^{13} \mathrm{C}-\mathrm{NMR}\left(100 \mathrm{MHz}, \mathrm{CDCl}_{3}\right): \delta 178.9,140.6,139.5,136.4,130.4,129.1,128.4,126.0,124.4,122.5,109.7$, 59.4, 33.5, 33.2, 28.1, 22.4, 22.2, 21.2; HRMS (ESI) $m$ /e calcd. for $\mathrm{C}_{20} \mathrm{H}_{23} \mathrm{NOS}(\mathrm{M}+\mathrm{H})^{+} 326.1573$, found 326.1570 .

3-Cyclohexyl-3-( $p$-tolylthio)indolin-2-one (3ga). After purification by silica gel column chromatography (PE:EA = 4:1), compound 3ga was isolated as a white solid $(56 \mathrm{mg}, 67 \%)$; m.p. $=216-217{ }^{\circ} \mathrm{C}$; $R_{\mathrm{f}}(\mathrm{PE}: \mathrm{EA}=3: 1)=0.47 ;{ }^{1} \mathrm{H}-\mathrm{NMR}\left(400 \mathrm{MHz}, d_{6}\right.$-DMSO $): \delta 10.2(\mathrm{~s}, 1 \mathrm{H}), 7.35(\mathrm{~d}, J=7.4 \mathrm{~Hz}, 1 \mathrm{H})$, $7.11(\mathrm{t}, J=7.3 \mathrm{~Hz}, 1 \mathrm{H}), 7.00-6.93(\mathrm{~m}, 5 \mathrm{H}), 6.58(\mathrm{~d}, J=7.6 \mathrm{~Hz}, 1 \mathrm{H}), 2.19(\mathrm{~s}, 3 \mathrm{H}), 2.04(\mathrm{~d}, J=12 \mathrm{~Hz}, 1 \mathrm{H})$, $1.96(\mathrm{~d}, J=11.8 \mathrm{~Hz}, 1 \mathrm{H}), 1.76(\mathrm{~d}, J=12.2 \mathrm{~Hz}, 1 \mathrm{H}), 1.59-1.53(\mathrm{~m}, 3 \mathrm{H}), 1.35-1.10(\mathrm{~m}, 3 \mathrm{H}), 1.04-0.97(\mathrm{~m}, 1 \mathrm{H})$, 0.86-0.76 (m, 1H); ${ }^{13} \mathrm{C}-\mathrm{NMR}$ (100 MHz, $d_{6}$-DMSO): $\delta 176.5,141.8,138.7,135.7,129.2,129.0,128.4,126.0$, 125.1, 121.4, 109.1, 63.2, 43.3, 27.6, 27.2, 25.9, 25.7, 25.7, 20.6; HRMS (ESI) $m / e$ calcd. for $\mathrm{C}_{21} \mathrm{H}_{23} \mathrm{NOS}$ $(\mathrm{M}+\mathrm{H})^{+}$338.1573, found 338.1572.

3-Cyclohexyl-3-((4-methoxyphenyl)thio)indolin-2-one (3gb). After purification by silica gel column chromatography (PE:EA = 5:1), compound $3 \mathrm{gb}$ was isolated as a white solid $(52 \mathrm{mg}, 65 \%)$; m.p. $=198-199{ }^{\circ} \mathrm{C} ; R_{\mathrm{f}}(\mathrm{PE}: \mathrm{EA}=3: 1)=0.42 ;{ }^{1} \mathrm{H}-\mathrm{NMR}\left(400 \mathrm{MHz}, \mathrm{CDCl}_{3}\right): \delta 7.76(\mathrm{~s}, 1 \mathrm{H})$, $7.45(\mathrm{~d}, J=7.3 \mathrm{~Hz}, 1 \mathrm{H}), 7.13(\mathrm{td}, J=7.6 \mathrm{~Hz}, 1.1 \mathrm{~Hz}, 1 \mathrm{H}), 7.08-7.04(\mathrm{~m}, 3 \mathrm{H}), 6.60(\mathrm{~d}, J=7.7 \mathrm{~Hz}$, $1 \mathrm{H}), 6.57(\mathrm{~d}, J=8.8 \mathrm{~Hz}, 2 \mathrm{H}), 3.68(\mathrm{~s}, 3 \mathrm{H}), 2.21(\mathrm{~m}, 2 \mathrm{H}), 1.83(\mathrm{~d}, J=12.6 \mathrm{~Hz}, 1 \mathrm{H}), 1.64(\mathrm{~d}, J=10.6 \mathrm{~Hz}, 2 \mathrm{H})$, $1.42-1.22(\mathrm{~m}, 4 \mathrm{H}), 1.13-0.88(\mathrm{~m}, 2 \mathrm{H}) ;{ }^{13} \mathrm{C}-\mathrm{NMR}\left(100 \mathrm{MHz}, \mathrm{CDCl}_{3}\right): \delta 179.0,160.4,140.7,137.9,129.9$, 128.3, 125.6, 122.2, 120.0, 113.7, 109.6, 64.3, 55.0, 43.7, 28.3, 27.8, 26.5, 26.2, 26.1; HRMS (ESI) m/e calcd. for $\mathrm{C}_{21} \mathrm{H}_{23} \mathrm{NO}_{2} \mathrm{~S}(\mathrm{M}+\mathrm{H})^{+} 354.1522$, found 354.1522 .

4-((2-Oxo-3-( $p$-tolylthio)indolin-3-yl)methyl)benzonitrile (3ha). After purification by silica gel column chromatography (PE:EA = 3:1), compound 3ha was isolated as a white solid $(56 \mathrm{mg}, 60 \%)$; m.p. $=238-239{ }^{\circ} \mathrm{C} ; R_{\mathrm{f}}(\mathrm{PE}: \mathrm{EA}=3: 1)=0.26 ;{ }^{1} \mathrm{H}-\mathrm{NMR}\left(400 \mathrm{MHz}, d_{6}\right.$-DMSO): $\delta 10.17(\mathrm{~s}, 1 \mathrm{H}), 7.56$ $(\mathrm{d}, J=8.3 \mathrm{~Hz}, 2 \mathrm{H}), 7.45(\mathrm{~d}, J=7.0 \mathrm{~Hz}, 1 \mathrm{H}), 7.11(\mathrm{~d}, J=8.2 \mathrm{~Hz}, 4 \mathrm{H}), 7.07-7.03(\mathrm{~m}, 3 \mathrm{H}), 7.00(\mathrm{td}, J=7.5 \mathrm{~Hz}$, $0.8 \mathrm{~Hz}, 1 \mathrm{H}), 6.44(\mathrm{~d}, J=7.5 \mathrm{~Hz}, 1 \mathrm{H}), 3.52(\mathrm{~d}, J=12.9 \mathrm{~Hz}, 1 \mathrm{H}), 3.35(\mathrm{~d}, J=12.9 \mathrm{~Hz}, 1 \mathrm{H}), 2.24(\mathrm{~s}, 3 \mathrm{H})$; ${ }^{13} \mathrm{C}-\mathrm{NMR}\left(100 \mathrm{MHz}, d_{6}\right.$-DMSO): $\delta$ 175.8, 141.3, 141.2, 139.4, 136.1, 131.6, 130.9, 129.2, 128.9, 128.1, 125.6, 125.0, 121.5, 118.5, 109.6, 109.4, 59.2, 39.9, 20.7; HRMS (ESI) $m / e$ calcd. for $\mathrm{C}_{23} \mathrm{H}_{18} \mathrm{~N}_{2} \mathrm{OS}(\mathrm{M}+\mathrm{H})^{+}$ 371.1212 , found 371.1213 .

3-(4-Chlorobenzyl)-3-(p-tolylthio)indolin-2-one (3ia). After purification by silica gel column chromatography (PE:EA = 5:1), compound 3ia was isolated as a white solid $(75 \mathrm{mg}, 79 \%$ ); 
m.p. $=217-218{ }^{\circ} \mathrm{C} ; R_{\mathrm{f}}(\mathrm{PE}: \mathrm{EA}=3: 1)=0.50 ;{ }^{1} \mathrm{H}-\mathrm{NMR}\left(400 \mathrm{MHz}, d_{6}\right.$-DMSO): $\delta 10.1(\mathrm{~s}, 1 \mathrm{H}), 7.42$ $(\mathrm{d}, J=7.1 \mathrm{~Hz}, 1 \mathrm{H}), 7.13(\mathrm{~d}, J=8.4 \mathrm{~Hz}, 2 \mathrm{H}), 7.10(\mathrm{~d}, J=8.1 \mathrm{~Hz}, 2 \mathrm{H}), 7.07-7.02(\mathrm{~m}, 3 \mathrm{H}), 6.97(\mathrm{t}, J=6.7$ $\mathrm{Hz}, 1 \mathrm{H}), 6.92(\mathrm{~d}, J=8.4 \mathrm{~Hz}, 2 \mathrm{H}), 6.45(\mathrm{~d}, J=7.6 \mathrm{~Hz}, 1 \mathrm{H}), 3.40(\mathrm{~d}, J=13.0 \mathrm{~Hz}, 1 \mathrm{H}), 3.26(\mathrm{~d}, J=13.0 \mathrm{~Hz}$, 1H), 2.24 (s, 3H); ${ }^{13} \mathrm{C}-\mathrm{NMR}$ (100 MHz, $d_{6}$-DMSO): $\delta 176.1,141.5,139.4,136.1,134.4,131.7,131.5,129.0$, $128.8,128.5,127.8,125.8,125.05,121.5,109.3,59.4,39.5,20.8$; HRMS (ESI) m/e calcd. for $\mathrm{C}_{22} \mathrm{H}_{18} \mathrm{ClNOS}$ $(\mathrm{M}+\mathrm{H})^{+} 380.0870$, found 380.0870 .

3-( $p$-Tolyl)-3-( $p$-tolylthio)indolin-2-one (5aa). After purification by silica gel column chromatography (PE:EA $=5: 1)$, compound 5aa was isolated as a white solid $(37 \mathrm{mg}, 42 \%) ; \mathrm{m} . \mathrm{p} .=196-197{ }^{\circ} \mathrm{C} ; R_{\mathrm{f}}(\mathrm{PE}: \mathrm{EA}$ $=3: 1)=0.41 ;{ }^{1} \mathrm{H}-\mathrm{NMR}\left(400 \mathrm{MHz}, \mathrm{CDCl}_{3}\right): \delta 7.65(\mathrm{~s}, 1 \mathrm{H}), 7.59(\mathrm{~d}, J=8.2 \mathrm{~Hz}, 2 \mathrm{H}), 7.40(\mathrm{~d}, J=7.4 \mathrm{~Hz}$, $1 \mathrm{H}), 7.17(\mathrm{~d}, J=8.5 \mathrm{~Hz}, 2 \mathrm{H}), 7.16-7.10(\mathrm{~m}, 2 \mathrm{H}), 7.08(\mathrm{~d}, J=8.0 \mathrm{~Hz}, 2 \mathrm{H}), 6.87(\mathrm{~d}, J=7.9 \mathrm{~Hz}, 2 \mathrm{H}), 6.64$ $(\mathrm{d}, J=7.6 \mathrm{~Hz}, 1 \mathrm{H}), 2.34(\mathrm{~s}, 3 \mathrm{H}), 2.22(\mathrm{~s}, 3 \mathrm{H}) ;{ }^{13} \mathrm{C}-\mathrm{NMR}\left(100 \mathrm{MHz}, \mathrm{CDCl}_{3}\right): \delta 177.8,140.3,139.6,138.0$, 136.2, 133.2, 130.8, 129.3, 129.1, 128.6, 127.9, 126.5, 126.3, 122.5, 110.1, 62.8, 21.2, 21.0; HRMS (ESI) $\mathrm{m} / \mathrm{e}$ calcd. for $\mathrm{C}_{22} \mathrm{H}_{19} \mathrm{NOS}(\mathrm{M}+\mathrm{H})^{+} 346.1260$, found 346.1260 .

4-((2-Oxo-3-( $p$-tolyl)indolin-3-yl)thio)benzonitrile (5ai). After purification by silica gel column chromatography (PE:EA = 5:1), compound 5ai was isolated as a pale solid $(37 \mathrm{mg}, 41 \%) ; \mathrm{m} . \mathrm{p} .=178-181$ ${ }^{\circ} \mathrm{C} ; R_{\mathrm{f}}(\mathrm{PE}: \mathrm{EA}=3: 1)=0.36 ;{ }^{1} \mathrm{H}-\mathrm{NMR}\left(400 \mathrm{MHz}, \mathrm{CDCl}_{3}\right): \delta 7.64(\mathrm{~s}, 1 \mathrm{H}), 7.56(\mathrm{~d}, J=8.3 \mathrm{~Hz}, 2 \mathrm{H})$, $7.42(\mathrm{~d}, J=7.5 \mathrm{~Hz}, 1 \mathrm{H}), 7.35(\mathrm{~d}, J=8.4 \mathrm{~Hz}, 2 \mathrm{H}), 7.30(\mathrm{~d}, J=8.4 \mathrm{~Hz}, 2 \mathrm{H}), 7.24-7.19(\mathrm{~m}, 3 \mathrm{H}), 7.12$ $(\mathrm{t}, J=7.6 \mathrm{~Hz}, 1 \mathrm{H}), 6.71(\mathrm{~d}, J=7.7 \mathrm{~Hz}, 1 \mathrm{H}), 2.35(\mathrm{~s}, 3 \mathrm{H}) ;{ }^{13} \mathrm{C}-\mathrm{NMR}\left(100 \mathrm{MHz}, \mathrm{CDCl}_{3}\right): \delta 177.0,139.9$, 138.7, 137.1, 135.5, 132.3, 131.7, 129.8, 129.6, 129.35, 127.72, 126.3, 123.0, 118.2, 112.5, 110.3, 62.6, 21.1; HRMS (ESI) $m / e$ calcd. for $\mathrm{C}_{22} \mathrm{H}_{16} \mathrm{~N}_{2} \mathrm{OS}(\mathrm{M}+\mathrm{H})^{+} 357.1056$, found 357.1058.

3-((3-Chlorophenyl)thio)-3-( -tolyl)indolin-2-one (5ah). After purification by silica gel column chromatography (PE:EA $=5: 1)$, compound 5 ah was isolated as a white solid $(65 \mathrm{mg}, 71 \%)$; m.p. $=192-193{ }^{\circ} \mathrm{C} ; R_{\mathrm{f}}(\mathrm{PE}: \mathrm{EA}=3: 1)=0.40 ;{ }^{1} \mathrm{H}-\mathrm{NMR}\left(400 \mathrm{MHz}, \mathrm{CDCl}_{3}\right): \delta 7.68(\mathrm{~s}, 1 \mathrm{H}), 7.58(\mathrm{~d}, J=8.2 \mathrm{~Hz}$, $2 \mathrm{H}), 7.43(\mathrm{~d}, J=7.3 \mathrm{~Hz}, 1 \mathrm{H}), 7.22-7.12(\mathrm{~m}, 5 \mathrm{H}), 7.19(\mathrm{~d}, J=7.8 \mathrm{~Hz}, 2 \mathrm{H}), 7.00(\mathrm{t}, J=7.9 \mathrm{~Hz}, 1 \mathrm{H})$, $6.68(\mathrm{~d}, J=7.6 \mathrm{~Hz}, 1 \mathrm{H}), 2.35(\mathrm{~s}, 3 \mathrm{H}) ;{ }^{13} \mathrm{C}-\mathrm{NMR}\left(100 \mathrm{MHz}, \mathrm{CDCl}_{3}\right): \delta 177.3,140.1,138.4,135.7,134.1$, $133.7,132.5,131.9,130.1,129.6,129.4,129.3,129.1,127.9,126.4,122.8,110.2,62.8,21.1 ;$ HRMS (ESI) $m / e$ calcd. for $\mathrm{C}_{21} \mathrm{H}_{16} \mathrm{ClNOS}(\mathrm{M}+\mathrm{H})^{+} 366.0713$, found 366.0711.

5-Bromo-3-( $p$-tolyl)-3-( $p$-tolylthio)indolin-2-one (5ba). After purification by silica gel column chromatography (PE:EA $=3: 1)$, compound 5 ba was isolated as a white solid $(51 \mathrm{mg}, 48 \%$ ); m.p. $=213-215{ }^{\circ} \mathrm{C} ; R_{\mathrm{f}}(\mathrm{PE}: \mathrm{EA}=3: 1)=0.32 ;{ }^{1} \mathrm{H}-\mathrm{NMR}\left(400 \mathrm{MHz}, \mathrm{CDCl}_{3}\right): \delta 7.99(\mathrm{~s}, 1 \mathrm{H}), 7.54(\mathrm{~d}, J=8.4 \mathrm{~Hz}$, $2 \mathrm{H}), 7.43(\mathrm{~d}, J=2.0 \mathrm{~Hz}, 1 \mathrm{H}), 7.28(\mathrm{dd}, J=8.4 \mathrm{~Hz}, 2.0 \mathrm{~Hz}, 1 \mathrm{H}), 7.19(\mathrm{~d}, J=8.4 \mathrm{~Hz}, 2 \mathrm{H}), 7.09(\mathrm{~d}, J=8.0$ $\mathrm{Hz}, 2 \mathrm{H}), 6.91(\mathrm{~d}, J=7.9 \mathrm{~Hz}, 2 \mathrm{H}), 6.55(\mathrm{~d}, J=8.3 \mathrm{~Hz}, 1 \mathrm{H}), 2.35(\mathrm{~s}, 3 \mathrm{H}), 2.23(\mathrm{~s}, 3 \mathrm{H}) ;{ }^{13} \mathrm{C}-\mathrm{NMR}(100 \mathrm{MHz}$, $d_{6}$-DMSO): $\delta 175.2,140.5,139.7,137.8,135.7,133.0,132.5,131.7,129.4,129.3,128.5,127.5,126.2,113.4$, $111.8,62.2,20.8$, 20.7; HRMS (ESI) m/e calcd. for $\mathrm{C}_{22} \mathrm{H}_{18} \operatorname{BrNOS}(\mathrm{M}+\mathrm{H})^{+} 424.0365$, found 424.0363.

3-(3-Methoxyphenyl)-3-(p-tolylthio)indolin-2-one (5ca). After purification by silica gel column chromatography (PE:EA $=5: 1)$, compound 5 ca was isolated as a white solid $(35 \mathrm{mg}, 39 \%)$; m.p. $=175-176{ }^{\circ} \mathrm{C} ; R_{\mathrm{f}}(\mathrm{PE}: \mathrm{EA}=3: 1)=0.33 ;{ }^{1} \mathrm{H}-\mathrm{NMR}\left(400 \mathrm{MHz}, \mathrm{CDCl}_{3}\right): \delta 7.93(\mathrm{~s}, 1 \mathrm{H}), 7.40(\mathrm{~d}, J=7.3 \mathrm{~Hz}$, $1 \mathrm{H}), 7.31-7.11(\mathrm{~m}, 4 \mathrm{H}), 7.11-7.07(\mathrm{~m}, 3 \mathrm{H}), 6.86(\mathrm{~d}, J=7.6 \mathrm{~Hz}, 3 \mathrm{H}), 6.66(\mathrm{~d}, J=7.6 \mathrm{~Hz}, 1 \mathrm{H}), 3.81(\mathrm{~s}, 3 \mathrm{H}), 2.21$ (s, 3H); ${ }^{13} \mathrm{C}-\mathrm{NMR}\left(100 \mathrm{MHz}, \mathrm{CDCl}_{3}\right): \delta 177.1,159.7,140.1,139.8,137.7,136.2,130.6,129.5,129.2,128.7$, 126.4, 122.6, 120.4, 114.1, 113.7, 109.9, 62.8, 55.3, 55.3, 21.2; HRMS (ESI) m/e calcd. for $\mathrm{C}_{22} \mathrm{H}_{19} \mathrm{NO}_{2} \mathrm{~S}$ $(\mathrm{M}+\mathrm{H})^{+}$362.1209, found 362.1208.

\section{Conclusions}

We have developed a new synthesis of oxindole thioethers by triphenylphosphine-mediated deoxygenation-thiolation of oxindoles with sulfonyl chlorides as sulfenylation reagents. The above reaction was facilitated by iodide anions, possibly due to the in situ conversion of sulfenyl chlorides to the more reactive sulfenyl iodides. Sulfenylation of 3-aryloxindoles required the presence of a base. The use of a transition-metal-free protocol, readily available reagents, and mild reaction conditions 
allow this protocol more practical to prepare 3-sulfanyloxindoles than traditional methods. This study demonstrated the potential of sulfonyl chlorides as novel, readily accessible, and environmentally friendly sulfenylation reagents for direct thiolation of electron-rich heterocycles.

Supplementary Materials: The following are available online, ${ }^{1} \mathrm{H}-\mathrm{NMR}$ and ${ }^{13} \mathrm{C}-\mathrm{NMR}$ of compound $3 \mathbf{a a}-\mathbf{3 g b}$ and 5aa-5ca.

Acknowledgments: The authors sincerely thank the financial support from National Science Foundation of China (Grants 21572158).

Author Contributions: X.Z. and K.L. designed the experiments and wrote the paper; A.W. and X.L. performed the experiments and analyzed the data.

Conflicts of Interest: The authors declare no conflict of interest.

\section{References}

1. Pedras, M.S.C.; Yaya, E.E.; Glawischnig, E. The phytoalexins from cultivated and wild crucifers: Chemistry and biology. Nat. Prod. Rep. 2011, 28, 1381-1405. [CrossRef] [PubMed]

2. Millemaggi, A.; Taylor, R.J.K. 3-Alkenyl-oxindoles: Natural products, pharmaceuticals, and recent synthetic advances in tandem/telescoped approaches. Eur. J. Org. Chem. 2010, 4527-4547. [CrossRef]

3. Trost, B.M.; Brennan, M.K. Asymmetric syntheses of oxindole and indole spirocyclic alkaloid natural products. Synthesis 2009, 3003-3025. [CrossRef]

4. Galliford, C.V.; Scheidt, K.A. Pyrrolidinyl-spirooxindole natural products as inspirations for the development of potential therapeutic agents. Angew. Chem. Int. Ed. 2007, 46, 8748-8758. [CrossRef] [PubMed]

5. Marti, C.; Carreira, E.M. Construction of spiro[pyrrolidine-3, $3^{\prime}$-oxindoles]-recent applications to the synthesis of oxindole alkaloids. Eur. J. Org. Chem 2003, 2209-2219. [CrossRef]

6. Mehta, R.G.; Liu, J.; Constantinou, A.; Hawthorne, M.; Pezzuto, J.M.; Moon, R.C.; Moriarty, R.M. Structure-activity relationships of brassinin in preventing the development of carcinogen-induced mammary lesions in organ culture. Anticancer Res. 1994, 14, 1209-1213. [PubMed]

7. Pedras, M.S.C.; Hossain, M. Metabolism of crucifer phytoalexins in Sclerotinia sclerotiorum: Detoxification of strongly antifungal compounds involves glucosylation. Org. Biomol. Chem. 2006, 4, 2581-2590. [CrossRef] [PubMed]

8. Dandia, A.; Sati, M.; Arya, K.; Sharma, R.; Loupy, A. Facile one pot microwave induced solvent-free synthesis and antifungal, antitubercular screening of spiro [1,5]-benzothiazepin-2, $3^{\prime}\left[3^{\prime} \mathrm{H}\right]$ indol-2[1 $\left.1^{\prime} \mathrm{H}\right]$-ones. Chem. Pharm. Bull. 2003, 51, 1137-1141. [CrossRef] [PubMed]

9. Li, Y.; Shi, Y.; Huang, Z.; Wu, X.; Xu, P.; Wang, J.; Zhang, Y. Catalytic thia-sommelet-hauser rearrangement: Application to the synthesis of oxindoles. Org. Lett. 2011, 13, 1210-1213. [CrossRef] [PubMed]

10. Coote, S.C.; Quenum, S.; Procter, D.J. Exploiting Sm(II) and Sm(III) in $\mathrm{SmI}_{2}$-initiated reaction cascades: Application in a tag removal-cyclization approach to spirooxindole scaffolds. Org. Biomol. Chem. 2011, 9, 5104-5108. [CrossRef] [PubMed]

11. Miller, M.; Vogel, J.C.; Tsang, W.; Merrit, A.; Procter, D.J. Formation of N-heterocycles by the reaction of thiols with glyoxamides: Exploring a connective Pummerer-type cyclization. Org. Biomol. Chem. 2009, 7, 589-597. [CrossRef] [PubMed]

12. Shen, Y.; Atobe, M.; Fuchigami, T. Electroorganic synthesis using a fluoride ion mediator under ultrasonic irradiation: Synthesis of oxindole and 3-oxotetrahydroisoquinoline derivatives. Org. Lett. 2004, 6, 2441-2444. [CrossRef] [PubMed]

13. Liao, Y.J.; Wu, Y.L.; Chuang, C.P. Cyclization reactions of methylthioacetanilides. Tetrahedron 2003, 59, 3511-3520. [CrossRef]

14. Greaney, M.F.; Motherwell, W.B. Studies on the oxidation and fluorination of $\alpha$-(phenylsulfanyl) acetamides using (difluoroiodo) toluene. Tetrahedron. Lett. 2000, 41, 4467-4470. [CrossRef]

15. Al-thebeiti, M.S. Synthesis of some new spiro[indoline-3,2'-(thiochroman)]-2,4'-dione derivatives. Phosphoru Sulfur Silicon Relat. Elem. 1998, 141, 89-95. [CrossRef]

16. Young, S.D.; Amblard, M.C.; Britcher, S.F.; Grey, V.E.; Tran, L.O.; Lumma, W.C.; Huff, J.R.; Schleif, W.A.; Emini, E.E.; O'Brien, J.A. 2-Heterocyclic indole-3-sulfones as inhibitors of HIV-1 reverse transcriptase. Bioorg. Med. Chem. Lett. 1995, 5, 491-496. [CrossRef] 
17. Cai, Y.; Li, J.; Chen, W.; Xie, M.; Liu, X.; Lin, L.; Feng, X. Catalytic asymmetric sulfenylation of unprotected 3-substituted oxindoles. Org. Lett. 2012, 14, 2726-2729. [CrossRef] [PubMed]

18. Li, X.; Liu, C.; Xue, X.S.; Cheng, J.P. Enantioselective organocatalyzed sulfenylation of 3-substituted oxindoles. Org. Lett. 2012, 14, 4374-4377. [CrossRef] [PubMed]

19. Wu, Q.; Zhao, D.; Qin, X.; Lan, J.; You, J. Synthesis of di(hetero) aryl sulfides by directly using arylsulfonyl chlorides as a sulfur source. Chem. Commun. 2011, 47, 9188-9190. [CrossRef] [PubMed]

20. Chen, M.; Huang, Z.T.; Zheng, Q.Y. Visible light-induced 3-sulfenylation of N-methylindoles with arylsulfonyl chlorides. Chem. Commun. 2012, 48, 11686-11688. [CrossRef] [PubMed]

21. Zhao, X.; Lu, X.; Wei, A.; Jia, X.; Chen, J.; Lu, K. Potassium iodide promoted thiolation of pyrazolones and benzofurans using aryl sulfonyl chlorides as sulfenylation reagents. Tetrahedron Lett. 2016, 57, 5330-5333. [CrossRef]

22. Lu, K.; Deng, Z.; Li, M.; Li, T.; Zhao, X. Transition metal-free direct trifluoromethylthiolation of indoles using trifluoromethanesulfonyl chloride in the presence of triphenylphosphine. Org. Biomol. Chem. 2017, 15, 1254-1260. [CrossRef] [PubMed]

23. Zhao, X.; Wei, A.; Li, T.; Su, Z.; Chen, J.; Lu, K. Transition-metal free direct difluoromethylthiolation of electron-rich aromatics with difluoromethanesulfonyl chloride. Org. Chem. Front. 2017, 4, 232-235. [CrossRef]

24. Zhao, X.; Li, T.; Yang, B.; Qiu, D.; Lu, K. Transition-metal-free trifluoromethylthiolation and difluoromethylthiolation of thiols with trifluoromethanesulfonyl chloride and difluoromethanesulfonyl chloride. Tetrahedron 2017, 73, 3112-3117. [CrossRef]

25. Zhao, X.; Deng, Z.; Wei, A.; Li, B.; Lu, K. Iodine-catalysed regioselective thiolation of flavonoids using sulfonyl hydrazides as sulfenylation reagents. Org. Biomol. Chem. 2016, 14, 7304-7312. [CrossRef] [PubMed]

26. Zhao, X.; Li, T.; Zhang, L.; Lu, K. Iodine-catalyzed thiolation of electron-rich aromatics using sulfonyl hydrazides as sulfenylation reagents. Org. Biomol. Chem. 2016, 14, 1131-1137. [CrossRef] [PubMed]

27. Zhao, X.; Zhang, L.; Lu, X.; Li, T.; Lu, K. Synthesis of 2-aryl and 3-aryl benzo[b]furan thioethers using aryl sulfonyl hydrazides as sulfenylation reagents. J. Org. Chem. 2015, 80, 2918-2924. [CrossRef] [PubMed]

28. Zhao, X.; Zhang, L.; Li, T.; Liu, G.; Wang, H.; Lu, K. p-Toluenesulphonic acid-promoted, $\mathrm{I}_{2}$-catalysed sulphenylation of pyrazolones with aryl sulphonyl hydrazides. Chem. Commun. 2014, 50, 13121-13123. [CrossRef] [PubMed]

29. Dua, T.P.; Zhu, G.G.; Zhou, J. A facile method for the synthesis of 3-alkyloxindole. Lett. Org. Chem. 2012, 9, 225-232. [CrossRef]

Sample Availabilty: Samples of the compounds $\mathbf{3 a a}-\mathbf{3 g b}$ and $\mathbf{5 a a}-\mathbf{5 c a}$ are available from the authors. 